\title{
Efficacy of application of eutectic mixture of local anesthetics and lidocaine spray in pain management of arteriovenous fistula cannulation in hemodialysis patients
}

\author{
Samaneh Mirzaei ${ }^{1}$, Mostafa Javadi ${ }^{*}$, Adel Eftekhari ${ }^{2}$, Maryam Hatami ${ }^{3}$, Roya Hemayati ${ }^{4}$ \\ ${ }^{1}$ Department of Nursing, School of Nursing and Midwifery, Shahid Sadoughi University of Medical Sciences, Yazd, Iran \\ ${ }^{2}$ Department of Public Health, Shahid Sadoughi University of Medical Sciences, Yazd, Iran \\ ${ }^{3}$ Department of Anesthesiology, Shahid Sadoughi University of Medical Sciences, Yazd, Iran \\ ${ }^{4}$ Department of Internal Medicine, Shahid Sadoughi University of Medical Sciences, Yazd, Iran
}

\section{A R T I C L E I N F O}

Article Type:

Original

\section{Article History:}

Received: 10 February 2017

Accepted: 27 June 2017

Published online: 20 July 2017

\section{Keywords:}

Hemodialysis

Arteriovenous fistula cannulation Lidocaine

Pain

\begin{abstract}
A B S T RACT
Introduction: The hemodialysis patients frequently experience the pain and anxiety induced by arteriovenous fistula (AVF) cannulation. This painful intervention, if repeated, imposes destructive psychosomatic effects on patients. Hence, the use of appropriate procedures to reduce pain in these patients is of utmost importance.

Objectives: The present study aimed at investigate efficacy of lidocaine spray and topical eutectic mixture of local anesthetics (EMLA) cream in releiving pain induced by arteriovenous fistula cannulation in hemodialysis patients.

Patients and Methods: This quasi-experimental study was conducted on 40 patients with arteriovenous fistula (AVF) selected on the basis of purposive sampling method in 2015 in hemodialysis ward of Shahid-Sadoughi hospital. Pain severity was measured at AVF cannulation using EMLA analgesic cream and lidocaine spray with the pain severity numerical scale. Repeated measures analysis of variance (ANOVA) was used in data analysis using SPSS 16.

Results: Findings showed that the mean scores of the three methods of pain management, i.e., no pain control method, lidocaine spray, and EMLA analgesic cream, were $7.45 \pm 0.88$, $4.22 \pm 1.33$, and $2.8 \pm 0.70$, respectively. There was a considerable reduction in pain severity using the lidocaine spray and EMLA analgesic cream compared to the conventional method $(P<0.001)$. EMLA analgesic cream caused a greater reduction in pain rate compared to lidocaine spray $(P<0.001)$.

Conclusion: This study suggested that EMLA analgesic cream was more effective in reducing pain caused by AVF cannulation. Hence, it is recommended that the hemodialysis patients apply EMLA analgesic cream themselves at the time of the procedure to reduce the cannulation pain.
\end{abstract}

Implication for health policy/practice/research/medical education:

In the current study, we intended to measure pain associated with AVF cannulation and to compare the effectiveness of lidocaine spray and topical EMLA cream in controlling pain caused by venepuncture of AVF patients undergoing chronic hemodialysis. This study points out the need for an effective pain assessment before the AVF puncture, which can serve as a starting point for the elaboration of protocols for pain management in hemodialysis. We suggest experimental studies to compare different pharmacological and non-pharmacological approaches as alternatives to minimize pain.

Please cite this paper as: Mirzaei S, Javadi M, Eftekhari A. Efficacy of application of eutectic mixture of local anesthetics and lidocaine spray in pain management of arteriovenous fistula cannulation in hemodialysis patients. J Renal Inj Prev. 2017;6(4):269274. DOI: $10.15171 /$ jrip.2017.51. 


\section{Introduction}

The end-stage renal disease (ESRD) is the progressive irreversible degeneration of kidney functioning leading to pain and suffering for many people around the world. Figures and statistics suggest that the population of American hemodialysis patients is doubled every 10 years (1). It is estimated that the number of hemodialysis patients will exceed 3500000 by 2020 . The growth rate of this disorder is greater than the average global growth in Iran reaching about $12 \%$ per year (2). Hemodialysis as the most common treatment line for ESRD is a stressful process which may cause various psychosocial disturbances. These patients are usually affected with several complications some of which are related to ESRD and some others pertain to the type of its treatment (3). Among the complaints expressed by $50 \%$ of these patients is some sort of pain experience (4). Pain may induce disability, fear, and anxiety in most patients more than any other disease and it is one of the most common reasons for seeking healthcare and treatment by clients $(5,6)$. In the clinical setting, pain is produced during the performance of various diagnostic and therapeutic interventions among such as venous catheter or cannulation for therapeutic purposes in hospital (7). In hemodialysis patients, the most common cause of pain is the arteriovenous fistula (AVF) cannulation due to the diameter and length of these catheters (8). On average, the hemodialysis patients undergo the procedure three times a week, each time lasting 3-4 hours. Moreover, the hemodialysis patients averagely experience the cannulation pain and skin puncture 10 times per month and this pain continues during the lifespan of the patient or until a successful kidney graft is performed (9-11). The frequent pain induced by fistula cannulation in hemodialysis may result in depression, decreased life quality, pain and agony, distress, and stress in these patients while pain management may lead to the acceptance of hemodialysis by these patients and their improved life quality (12). Hence, reducing some part of the complications is of great importance for the longterm adaptation of the patients with hemodialysis (13). Regarding the significance of pain, pain relief should be considered as one part of treatment plan for these patients (14). Pain management is, in fact, an important part of nursing activities (15). Consequently, nurses should be aware of the physical and mental aspects of this pain and apply some effective strategies for managing it to improve the life quality of hemodialysis patients (7). Among the effective pharmaceutical methods of pain relief are the administration of topical anesthesia techniques such as the topical gel, anesthesia patch, and topical analgesic spray. These can reduce the pain induced by medical interventions like phlebotomy $(12,16)$. Lidocaine is one of the common and important agents used for local anesthesia (17). Lidocaine spray is one of the common forms of this agent used for clinical purposes with a moderate length of effect. It is used for the local anesthesia of mucous membranes and the skin. Depending on the site of interest, anesthesia is usually induced during 1-5 minutes and lasts 10-15 minutes (18). The pain relief theory behind lidocaine application is the blocking of active and inactive sodium channels followed by blocking of conduction and lack of stimulation resulting in reduced or impaired pain transmission (19). Another topical analgesic is topical eutectic mixture of local anesthetics (EMLA) cream which is a eutectic mixture of lidocaine $(2.5 \%)$ and prilocaine $(2.5 \%)$. It is used for various painful interventions on the skin (20). EMLA cream blocks the conduction of the electrical impulses through changing the depolarization of cellular membrane to sodium ions. This agent diffuses through intact skin providing analgesia in several millimeters of the superficial layers of the skin. The advantages of this agent include a localized action with little systemic absorption, easy administration, and its applicability by the patients themselves $(21,22)$. Many studies have been carried out on the effects of these two anesthetics (19). These studies demonstrated that the use of lidocaine components is effective in reducing cannulation pain (18). However, the results of the study by Turkmen et al revealed that $2.5 \%$ lidocaine had no effect on decreasing the pain of phlebotomy in children affected by chronic renal failure who were under hemodialysis (23).

\section{Objectives}

Regarding the controversial findings of these studies and also the significance of pain control in hemodialysis patients, this study aimed at determining the efficacy of lidocaine spray and EMLA analgesic cream in relieving the severity of cannulation pain in hemodialysis patients.

\section{Patients and Methods}

This quasi-experimental study was conducted on 40 hemodialysis patients presenting to the hemodialysis ward of Shahid Sadoughi hospital in Yazd, central Iran, in 2015. The intended sample who qualified for inclusion in the study entered the study after obtaining informed written consent. The required ethical issues were considered in the study. The researcher familiarized the participants with research purposes, methodology, voluntary participation in the study, and information confidentiality. The subjects were selected using the purposive sampling method. The inclusion criteria were; age $18+$ years, a history of at least three months of hemodialysis, being conscious, lack of sensitivity to lidocaine constituents, and lack of any problem in vascular accessibility. The data collection instruments included a questionnaire and a checklist consisting of two parts. The first part covered demographic information such as the patient's particulars like age, gender, marital status, occupation, history of present illness (HPI), education level, duration of hemodialysis, and the length of fistula use. The second part assessed the pain severity. Assessment of pain severity was performed using the pain severity numerical scale which was gauged from $0-10$. Zero indicated absence of any pain and 10 indicated the most severe pain experienced by the patient. The validity and reliability of the pain severity numerical 
scale was approved in several previous studies. The results of the study by Williamson and Hoggart revealed that this scale enjoyed acceptable validity and reliability coefficients and so it could be safely used in practical treatment (24). The pain severity at cannulation was assessed with three methods: the customary method in hemodialysis ward without pain management, the use of lidocaine spray, and the application of EMLA analgesic cream. Each patient was assessed three times for each method, i.e., a total of 9 assessments for each patient, while the order of the methods was determined randomly for each patient. To use the lidocaine spray method, two puffs ( $20 \mathrm{mg}$ ) of lidocaine were sprayed by the researcher on the skin surface from a $5-\mathrm{cm}$ distance near the cannulation site after prepping the skin. After 5 minutes, the cannulation site was disinfected with a cotton swab soaked in $70 \%$ alcohol and the special hemodialysis catheters were inserted into the AVF by the ward nurse. To administer the EMLA analgesic cream method, using a $2 \mathrm{~mL}$ syringe, $1.5 \mathrm{~g}$ of the EMLA cream was applied to the fistula site 20 minutes before the insertion of needle into the fistula site on a surface of almost $5 \mathrm{~cm}^{2}$ and fixed with supporting dressing. Then, the EMLA cream was removed, the site was disinfected with a cotton swab soaked in $70 \%$ alcohols, and special hemodialysis needles and catheters were inserted into the AVF by the ward nurse. For all patients and with all the three methods, pain severity was measured 2 minutes after the insertion of arteriovenous cannula using the pain severity numerical scale. All cannulations were performed with the same size needle (cannula), i.e., hemodialysis needle \# 16) by the ward nurse. If the cannulation was not performed successfully at the first attempt and skin puncture was repeated, the patient was excluded from the study.

\section{Ethical issues}

Sampling started after approval of the Committee of Ethics in Human Studies at Shahid Sadoughi University of Medical Sciences and permission of hospital authorities. The research followed the tenets of the Declaration of Helsinki. Informed consent was obtained; the research was approved by the ethical committee of Yazd University of Medical Sciences, Iran (ir.ssu.rec. 1394.85) and registered in Iranian Registry of Clinical Trials (http://www.irct.ir; Identifier: IRCT2015102224655N1).

\section{Statistical Analysis}

Statistical analysis was performed using SPSS (version 16) software package. In this study, descriptive statistics were used to analyze the demographic profiles of the participants. Repeated measure analysis of variance (ANOVA) test was used for the comparison of between the two interventions and for two-by-two comparisons using LSD post hoc test. A $P$ value $<0.05$ was considered significant.

\section{Results}

Of 42 patients who entered the study, two patients were excluded from the study due to their lack of cooperation and, consequently, 40 patients ultimately participated in the study (subject attrition =2). Of these, 25 patients (62.5\%) were male and 15 patients (37.5\%) were female. The mean age of the patients was 55.25 years. The mean length of hemodialysis in patients under study was $4.98 \pm 3.50$ years and the mean length of fistula stay was $3.74 \pm 2.63$ years. Furthermore, $92.5 \%$ of the patients were married while $7.5 \%$ were single. Also, $42.5 \%$ were illiterate, $32.5 \%$ had primary school education, and $25 \%$ had higher levels of education. Regarding history of hemodialysis-related diseases in the patients under study, our findings suggested that $87.5 \%$ had a positive history of hypertension, $50 \%$ a history of diabetes, $15 \%$ a history of cardiovascular diseases, and $2.5 \%$ a history of immunologic diseases. These findings demonstrated that hypertension by itself and in combination with diabetes was the most common cause of hemodialysis in the group under study. With respect to the goals of this study, the findings are shown in Tables 1 and 2.

Table 1 compares the fistula cannulation pain scores obtained for the conventional method without pain management, the lidocaine spray method, and the EMLA analgesic cream method in the patients under study. Using the repeated measure ANOVA, it was found that there was a significant difference among the mean scores of pain in the conventional method, the lidocaine spray method, and the EMLA analgesic cream method $(P<0.001)$.

Table 2 presents the two-by-two comparisons using LSD post hoc test. The values above the cut-off point relate to the absolute value of the difference between pain scores and the values below the cut-off point relate to the difference in statistical difference ( $P$ value) among the different methods. A two-by-two comparison of the methods revealed that the mean pain score was smaller using either the lidocaine spray or the EMLA analgesic cream compared to the conventional method without pain management $(P<0.001)$. Also, the pain mean score was smaller in EMLA analgesic cream compared to the lidocaine spray method with a statistically significant difference $(P<0.001$; Table 2$)$. This indicated a significant difference among the various methods.

Table 1. Pain scores of different methods before and after interventions

\begin{tabular}{lll}
\hline Method & Mean & Variance \\
\hline Without pain management & 7.45 & 0.88 \\
Spray lidocaine & 4.22 & 1.13 \\
EMLA analgesic cream & 2.80 & 0.70 \\
\hline
\end{tabular}

$P<0.001, F=289.1 ; d f=3$

Table 2. Presents the two-by-two comparisons using LSD post hoc test

\begin{tabular}{llll}
\hline & $\begin{array}{l}\text { Without pain } \\
\text { management }\end{array}$ & $\begin{array}{l}\text { Lidocaine } \\
\text { spray }\end{array}$ & $\begin{array}{l}\text { EMLA analgesic } \\
\text { cream }\end{array}$ \\
\hline $\begin{array}{l}\text { Without pain } \\
\text { management }\end{array}$ & - & 3.225 & 4.65 \\
Lidocaine spray & $P<0.001$ & - & 1.425 \\
EMLA analgesic cream & $P<0.001$ & $P<0.001$ & - \\
\hline
\end{tabular}




\section{Discussion}

This study was carried out to determine the efficacy of application of lidocaine spray and EMLA analgesic cream in reducing the AVF cannulation pain in hemodialysis patients. In this study, to remove the effect of intervening variables like age and gender. The research was conducted on one group using the conventional method without pain management, the lidocaine spray method, and the EMLA analgesic cream method in the hemodialysis ward. The findings of this study showed that most patients under study were male. In the study by Asgari et al on the effect of lidocaine spray on pain severity at hemodialysis cannulation, $63 \%$ of the patients were male and $37 \%$ were female (2). Moreover, in the study by Mohseni et al, $64 \%$ of the hemodialysis patients were male while $36 \%$ of them was female in each group (22). As can be inferred, the number of male hemodialysis patients is greater than the female patients in other similar studies. There are various reasons for the greater number of male hemodialysis patients compared to females. Raiesifar et al specified hypertension as the most common cause of renal failure in hemodialysis patients in their study conducted to investigate the causes of chronic renal failure in hemodialysis patients in Abadan, Iran (25). The study by Noblat et al demonstrated that the prevalence of renal failure following hypertension was higher in the male intensive care unit (ICU) in-patients compared to the females (26). Hence, regarding the causes contributing to the incidence of chronic renal failure and the results of the related studies, the greater number of male hemodialysis patients in the present study is justified. In addition, our findings showed that the mean age of the patients under study was 55.25 years. In the study by Mohseni et al, 72\% of the patients were $50+$ years old (22). In the study of Namadi and Movahedpour, the mean age of the patients was 55.05 years (2). As regard the most common cause of kidney disease is diabetes and hypertension, the prevalence of these diseases increases with age increasing (27). Hence, the prevalence of chronic renal failure aggravates with increasing age so that most patients afflicted with chronic renal failure are at higher ages. The findings of this study showed that the mean pain scores of the three methods, i.e., conventional method without pain management, the lidocaine spray method, and the EMLA analgesic cream method were $7.45 \pm 0.88,4.22 \pm 1.33$, and $2.80 \pm 0.70$, respectively. These findings suggested that the application of EMLA analgesic cream and lidocaine spray before fistula cannulation in hemodialysis patients reduced the cannulation pain significantly compared to the conventional method without pain management with a significant difference $(P<0.001)$. Also, our findings demonstrated that, comparing the two methods of lidocaine spray and EMLA analgesic cream, the reduction in pain severity was greater with EMLA analgesic cream compared to lidocaine spray indicating that EMLA cream decreases pain more effectively than lidocaine spray with a significant difference $(P<0.001)$. Various studies have been conducted so far on the use of various forms and combinations of lidocaine in controlling the sensation of pain in painful medical interventions. The study by Cuomo et al, to compare of the impact of $10 \%$ lidocaine spray on reducing the surgical debridement pain in venous ulcers in foot, showed that both interventions reduced pain score with no significant difference between the two, however, the patients in the lidocaine spray group needed more analgesics compared to the EMLA cream group. This finding indicated the greater effect of EMLA cream on pain reduction (28). These findings confirm the results of the present study. Additionally, the study by Asgari et al to detect, the effect of lidocaine spray on pain intensity at fistula cannulation in hemodialysis individuals, found no significant difference between pain severity in the two methods of lidocaine spray and placebo (2). Additionally, Mohseni et al compared the effects of topical piroxicam and EMLA analgesic cream on fistula cannulation pain severity to placebo in hemodialysis patients. They concluded that EMLA reduced the cannulation pain more significantly than either the topical piroxicam cream or the placebo $(P<0.001)(22)$. The results of other studies also indicated that mean pain severity is smaller with the administration of various forms of lidocaine compared to placebo. In this regard, the study by Benini et al, conducted to investigate the effect of EMLA cream on managing AVF cannulation pain in hemodialysis patients, showed that the analgesic cream was more effective in reducing fistula cannulation pain. They also stated that the effect of uncontrollable factors in the incidence of severe pain cannot be ignored (29). The study by Nott and Peacock conducted on 120 patients investigated the effects of EMLA cream on pain reduction in adult phlebotomy, showing the phlebotomy pain decreased significantly even 5 minutes after EMLA cream application (30); This finding is consistent with our results. Contrary to our findings, the results of the study by Turkmen et al, conducted on the effects of $2.5 \%$ lidocaine cream on pain reduction at pediatric phlebotomy in children with chronic renal failure under long-term hemodialysis demonstrated that $2.5 \%$ lidocaine had no significant effect on hemodialysis fistula cannulation pain; this finding is not consistent with ours. Obviously, they asserted that along with the use of topical analgesics, other factors like psychological parameters, skin puncture technique, and cannula or catheter size should also be considered (23). Contrary to our results, Qane et al determined the effect of EMLA cream on lumbar puncture pain, indicating no efficacy of this cream in pain reduction. They attributed the difference in their findings to the absence of EMLA cream supporting dressing and concluded that the use of supporting dressing could probably affect the rate of pain relief with EMLA cream (31). In general, cannulation stimulates the A delta and $\mathrm{C}$ receptors and this stimulation is transmitted via pain nervous fibers. Hence, the pain could be reduced by blocking the receptors stimulation or prevention of stimulation transmission at cannulation (2). Transmission of stimulation and the messaging function of the nervous system is mainly carried out by the potential 
changes in the neural membranes. Increased penetrability of sodium ion is created by nerve stimulation leading to accessibility of threshold potential and cellular depolarization. If the sodium current is blocked in the pathway of the pain-transmitting nerve fiber, transmission of neural message would be impossible. Lidocaine, in fact, blocks the transmission of the neural message by inhibiting sodium ion channels (32). In this study, the little time needed for the onset of effect of lidocaine spray ( 5 minutes) was considered as one of the important advantages of this spray affecting the speeding up of patients' affairs. In the study by Rogers and Ostrow, EMLA cream readily diffused into the epidermis due to its low melting point and blocked the initiation and conduction of the electrical impulse by changing the depolarization of the cellular membrane (23). Some findings suggested that EMLA analgesic cream penetrates through the intact skin inducing analgesia in superficial layers of skin in a thickness of several millimeters and reduces the sensation of pain in this way, approving the findings of the present study. The advantages of the application of EMLA cream in this study include localized action of EMLA cream with little systemic absorption, easy administration, and its applicability by the patients themselves. Also, the findings of some studies indicated that the hemodialysis patients prefer the topical anesthetic factors containing lidocaine over its parenteral application. In this respect, the study by Watson et al, conducted on the effects of local (topical) anesthetics on fistula cannulation pain in hemodialysis patients, reported that the hemodialysis patients preferred the use of lidocaine-containing topical cream over the parenteral lidocaine (33).

\section{Conclusion}

On the whole, the findings of this study on pain mean score showed that the AVF cannulation pain is a painful procedure in research subjects so that the patients experienced severe bothering pain at fistula cannulation when the conventional method was used without pain management. Based on the findings of the present study and those of similar studies, it could be concluded that both the lidocaine spray method and the EMLA analgesic cream method are effective in decreasing the fistula cannulation pain in hemodialysis patients. Hence, a painless anxiety-free experience could be created for these patients by training the personnel and patients in this regard. Nonetheless, the overall findings of this study indicate that the topical application of EMLA cream is more effective in decreasing the pain score of fistula cannulation. Therefore, regarding the severity of cannulation pain, this method could be used easily by the patients themselves as a self-administered technique.

\section{Limitations of the study}

There are some potential limitations to our study. The patients' exhaustion and distress as well as their initial resistance to participation may affect the study results. Another limitation is related to inability to blind of interventions that are beyond the control of researchers.

\section{Acknowledgements}

This paper was extracted from an MSc thesis of ICU Nursing at Shahid Sadoughi University of Medical Sciences based on a research project. Thus, the researchers would like to give special thanks to the university authorities for their financial support and approval by the Committee of Ethics at the university. Also, our thanks should go to the hard-working personnel of the hemodialysis ward at Shahid Rahnemoon hospital in Yazd, Iran who helped us in data collection processes. Finally, we should thank all the hemodialysis patients who voluntarily participated in the study.

\section{Authors' contribution}

SM; the concept, design, data analysis, and manuscript preparation. MJ; Study design, statistical analysis, manuscript editing, and manuscript review. AE; data collection. $\mathrm{RH}$ and $\mathrm{MH}$; Study consultants.

\section{Conflicts of interest}

The authors declared no competing interests.

\section{Ethical considerations}

Ethical issues (including data fabrication, double publication, and plagiarism) have been completely observed by the authors.

\section{Funding/Support}

This study was supported by the Deputy of Research of Shahid-Sadoughi University of Medical Sciences (Thesis \#2016).

\section{References}

1. Collins AJ, Foley RN, Gilbertson DT, Chen SC. The state of chronic kidney disease, ESRD, and morbidity and mortality in the first year of dialysis. Clin J Am Soc Nephrol. 2009;4:511. doi: 10.2215/CJN.05980809.

2. Namadi M, Movahedpour A. Quality of life in patients after renal transplantation in comparison with intermittent hemodialysis. J Ardabil Univ Med Sci. 2009;9:171-9. (Persian)

3. Azimian J, Abbasali Madadi Z, Falahatpishe F, Alipour Heidari M. Effect of warm footbath on arteriovenous fistula puncture-related pain in dialysis patients. J Qazvin Univ Med Sci. 2015;18:98-28. [Persian]

4. Davison SN. Pain in hemodialysis patients: prevalence, cause, severity, and management. Am J Kidney Dis. 2003;42:1239-47. doi: 10.1053/j.ajkd.2003.08.025.

5. Davison SN, Jhangri GS. Impact of pain and symptom burden on the health-related quality of life of hemodialysis patients. J Pain Symptom Manage. 2010;39:477-85. doi: 10.1016/j.jpainsymman.2009.08.008.

6. Nusstein J, Burns Y, Reader A, Beck M, Weaver J. Injection pain and postinjection pain of thepalatal-anterior superior alveolar injection, administered with the Wand Plus system, comparing 2\% lidocaine with 1:100,000 epinephrine to $3 \%$ mepivacaine. Oral Surg Oral Med Oral Pathol Oral Radiol Endod. 2004;97:164-72. doi:10.1016/S1079210403005766. 
7. Gong L, Liu J, Yan J, Wang L. Effect of puncture-related pain on the quality of life in patients undergoing maintenance hemodialysis through internal arteriovenous fistula. Zhong Nan Da Xue Xue Bao Yi Xue Ban. 2014;39:1292-8. doi: 10.11817/j.issn.1672-7347.2014.12.012.

8. Figueiredo AE, Viegas A, Monteiro M, Poli-de-Figueiredo CE. Research into pain perception with arteriovenous fistula (avf) cannulation. J Ren Care. 2008;34:169-72. doi: 10.1111/j.1755-6686.2008.00041.x.

9. PB S, Khakha DC, Mahajan S, Gupta S, Agarwal M, Yadav SL. Effect of cryotherapy on arteriovenous fistula puncturerelated pain in hemodialysis patients. Indian J Nephrol. 2008;18:155-8. doi: 10.4103/0971-4065.45290

10. Bagheri-Nesami M, Espahbodi F, Nikkhah A, Shorofi SA, Charati JY. The effects of lavender aromatherapy on pain following needle insertion into a fistula in hemodialysis patients. Complement Ther Clin Pract. 2014;20:1-4. doi: 10.1016/j.ctcp.2013.11.005.

11. Celik G, Ozbek O, Yilmaz M, Duman I, Ozbek S, Apiliogullari S. Vapocoolantspray vs lidocaine/prilocaine cream for reducing the pain of venipuncture in hemodialysis patients: a randomized, placebo-controlled, crossover study. Int J Med Sci. 2011;8:623-7.

12. Hassan A, Darwish MM, El-Samman GA, Fadel FI. The impact of cryotherapy on pain intensity at puncture sites of arteriovenous fistula among children undergoing hemodialysis. J Am Sci. 2012;8:12.

13. Sabitha $\mathrm{P}$, Khakha D, Mahajan S, Gupta S, Agarwal M, Yadav S. Effect of cryotherapy on arteriovenous fistula puncture-related pain in hemodialysis patients. Indian J Nephrol. 2008;18:155. doi: 10.4103/0971-4065.45290.

14. Park JS. The effect of cutaneous stimulation on AV fistula puncture pain of hemodialysis patients. Taehan Kanho. 1994;33:37-51.

15. Zyga S, Alikari V, Sachlas A, Stathoulis J, Aroni A, Theofilou $\mathrm{P}$, et al. Management of pain and quality of life in patients with chronic kidney disease undergoing hemodialysis. Pain Manag Nurs. 2015;16:712-20. doi: 10.1016/j. pmn.2015.03.004.

16. Hijazi R, Taylor D, Richardson J. Effect of topical alkane vapocoolant spray on pain with intravenous cannulation in patients in emergency departments: randomised double blind placebo controlled trial. BMJ. 2009;338:b215. doi: 10.1136/bmj.b215.

17. Marks DM, Newhouse A. Durability of benefit from repeated intravenous lidocaine infusions in fibromyalgia patients: a case series and literature review. Prim Care Companion CNS Disord. 2015;17. doi: 10.4088/PCC.15br01804.

18. Railan D, Alster TS. Use of topical lidocaine for cosmetic dermatologic procedures. J Drugs Dermatol. 2007;6:1104-8.

19. McNaughton C, Zhou C, Robert L, Storrow A, Kennedy R. A randomized, crossover comparison of injected buffered lidocaine, lidocaine cream, and no analgesia for peripheral intravenous cannula insertion. Ann Emerg Med.
2009;54:214-20. doi: 10.1016/j.annemergmed.2008.12.025.

20. Farnia F, Zeighamian S, Dehghani K, Lotfi H. Comparison of the effect of emla cream and cold compress on the Pain Intensity of Enoxaparin injection. Community Health Journal. 2014;8:46-39.

21. Irsfeld S, Klement W, Lipfert P. Dermal anaesthesia: comparison of EMLA cream with iontophoretic local anaesthesia. Br J Anaesth. 1993;71:375-8.

22. Mohseni M, Malekshahi F, Hadian B, Ebrahim Zadeh F. A comparison between the effects of topical piroxicam and EMLA cream on fistula cannulation pain in hemodialysis patient. Yafteh. 2015;16:110-2. [Persian]

23. Turkmen M, Kavukcu S, Soylu A, Ay N, Sevinc N, Sersan R. Topical anaesthesia in haemodialysis: evaluation of topical anaesthesia with lidocaine during vascular access in children undergoing long-term haemodialysis for chronic renal failure. Int J Nurs Pract. 1997;3:79-83

24. Williamson A, Hoggart B. Pain: a review7 of three commonly used pain rating scales. J Clin Nurs. 2005;14:798-804. doi: 10.1111/j.365-2702.005.01121.x.

25. Raiesifar A, Torabpour M, Mohsenizad P. Causes of chronic renal failure in hemodialysis patients of Abadan. Iran J Crit Care Nurs. 2010;2:11-2.

26. Noblat AC, Lopes MB, Lopes GB, Lopes AA. Complications of hypertension in men and women seen in a referral outpatient care unit. Arq Bras Cardiol. 2004;83:314-9.

27. Asgari M, Soleimani M. Comprehensive book Intensive nursing cares in CCU, ICU, and Dialysis wards. edt Boshra,2012; 391-393.

28. Cuomo R, D’Aniello C, Grimaldi L, Nisi G, Botteri G, Zerini I, et al. EMLA and lidocaine spray: a comparison for surgical debridement in venous leg ulcers. Adv Wound Care. 2015;4:358-61 doi: 10.1089/wound.2014.0605.

29. Benini F, Gobber D, Lago P, Agosto C, Carli G, Zacchello F. Pain management of arteriovenous fistula cannulation in haemodialysis children: efficacy of EMLA anaesthetic cream. Eur J Pain. 1998;2:109-13.

30. Nott MR, Peacock JL. Relief of injection pain in adults. EMLA cream for 5 minutes before venepuncture. Anaesthesia. 1990;45:772-4.

31. Qane M, Ghiliyan R, Hashemi A, Mirmohammadi S, Keshavarzi S, Yadegar Y, et al. The efficacy of the EMLA analgesic cream in compare with placebo in pediatric oncology lumbar puncture. Iranian Journal of Pediatric Hematology \& Oncology. 2012;2:44-8.

32. Haghighat A, Davoudi A, Minaiyan M, Molai M, Afshar A, Basiri K. Effect of a trial pharmaceutical solution on reversing sensations after using lidocain: An animal study. Anesth Essays Res. 2015;9:79-82. doi: 10.4103/02591162.150182 .

33. Watson AR, Szymkiw P, Morgan AG. Topical anaesthesia for fistula cannulation in hemodialysis patients. Nephrol Dial Transplant. 1988;3:800-2.

Copyright $\odot 2017$ The Author(s); Published by Nickan Research Institute. This is an open-access article distributed under the terms of the Creative Commons Attribution License (http://creativecommons.org/licenses/by/4.0), which permits unrestricted use, distribution, and reproduction in any medium, provided the original work is properly cited. 